further worsen survival in these patients and therefore prophylaxis against bleeding may not offer a survival advantage in these patients, who should be considered early for transplantation. The apparent importance of GOV in terms of prognosis, and decision making to optimise outcome means that we should re-look at strategies to screen for GOV in PBC.

\section{P37 PLATELET COUNT AND SPLEEN SIZE: AVOIDANCE OF SCREENING ENDOSCOPY FOR VARICES IN PATIENTS WITH HEPATITIS C CIRRHOSIS}

doi:10.1136/gutjnl-2011-300857a.37

\begin{abstract}
${ }^{1} J$ Gerada, ${ }^{2} E$ Gerada, ${ }^{3}$ S Sen, ${ }^{2}$ W J Griffiths. ${ }^{1}$ Addenbrookes' Hospital, Cambridge;
\end{abstract} ${ }^{2}$ Addenbrookes' Hospital; ${ }^{3}$ Cambridge

Introduction Many patients with liver cirrhosis, screened for oesophageal varices (OV) are found to have either no or insignificant varices, resulting in unnecessary procedures and financial burden. We had previously shown that the non-invasive parameters of platelet count and spleen size, measured by transabdominal ultrasound, were highly sensitive for the prediction of $\mathrm{OV}$ in patients with hepatitis $\mathrm{C}$ cirrhosis.

Aim To determine in a subsequent cohort of patients with hepatitis $\mathrm{C}$ cirrhosis whether such a clinical tool was accurate in determining which patients require endoscopic screening.

Method 246 outpatients with biopsy-proven hepatitis C cirrhosis over a 3 -year period were studied retrospectively. Endoscopy should have been performed, according to our protocol, if any of the following parameters were met: platelet count $<100 \times 10^{9} / 1$, spleen size _ $14 \mathrm{~cm}$ or specific ultrasound findings indicative of portal hypertension (recanalised paraumbilical vein, ascites, splenic hilar varices).

Results Of 246 patients, 98 patients underwent upper GI endoscopy, 76 of whom met criteria (78\%). Of 148 patients who did not undergo endoscopy, 63 met criteria (43\%). Endoscopic findings are summarised in the Abstract P37 table 1 below.

\section{Abstract P37 Table 1 Endoscopic findings}

\begin{tabular}{lllll}
\hline & OGD & & & \\
\cline { 2 - 3 } & $\begin{array}{l}\text { Significant } \\
\text { varices } \\
\text { (grade 2 } \\
\text { or more) }\end{array}$ & $\begin{array}{l}\text { Significant } \\
\text { varices } \\
\text { (grade 1 } \\
\text { or nil) }\end{array}$ & Predicted value & \\
\hline $\begin{array}{lllll}\text { Platelet count/ } \\
\text { spleen size/ }\end{array}$ & $\begin{array}{llll}\text { Criteria met } \\
\text { US findings }\end{array}$ & 14 & 62 & $14 / 76=18 \%$, PPV \\
& & 0 & 62 & $22 / 22=100$, NPV \\
& & $\begin{array}{l}14 / 14=100 \%, \\
\text { Criteria not met }\end{array}$ & $\begin{array}{l}\text { 22/84=26\%, } \\
\text { Specific }\end{array}$ & \\
\hline
\end{tabular}

Conclusion Using simple non-invasive criteria, screening endoscopy could be avoided in around 44\% of patients with hepatitis $\mathrm{C}$ cirrhosis. Treatable varices would not be missed in this group.

\section{P38 HOMODYNAMIC EFFECTS OF PROLONGED TREATMENT WITH MIDODRINE IN NON-AZOTEMIC PRE-ASCITIC, ASCITIC AND REFRACTORY ASCITES CIRRHOTIC PATIENTS}

\section{doi:10.1136/gutjnl-2011-300857a.38}

${ }^{1} \mathrm{H}$ Badran, ${ }^{2} \mathrm{~A}$ Moaty, ${ }^{3} \mathrm{~A}$ A Basuni, ${ }^{4}$ W F A Aziz, ${ }^{5} \mathrm{E}$ A Rewisha, ${ }^{5}$ A Waked. ${ }^{1}$ Tropical medicine; ${ }^{2}$ Hepatology; ${ }^{3}$ Biochemistry; ${ }^{4}$ Cardiology; ${ }^{5}$ internal medicine

Introduction Splanchnic arterial vasodilatation has related to hyperdynamic circulation and impaired natriuresis in advanced cirrhosis and was suggested to be responsible for the subtle sodium retention in pre-ascitic cirrhosis. $\alpha$ Adrenergic agonist may reverse this condition.
Aim This study aimed to evaluate the effects of treatment with the $\alpha 1$-adrenergic agonist midodrine on systemic haemodynamics in non-azotemic cirrhotic patients.

Method 154 cirrhotic patients were studied. The patients were classified into: absent ascites, mild to moderate diuretic responsive ascites and refractory ascites. Patients were randomly selected to receive either oral midodrine $10 \mathrm{mg}$, three times a day or placebo The following parameters were assessed for all patients: mean arterial pressure (MAP), cardiac output (CO), plasma rennin (PI R) and renal resistive index (RRI) at baseline, 7 days after administration of oral midodrine $10 \mathrm{mg}$, three times daily, and 3 months after administration of oral midodrine $2.5 \mathrm{mg}$, three times a day.

Results Midodrine administration induced a significant increase in MAP mention levels and $\mathrm{P}$ and significant decreases in $\mathrm{CO}$ mention levels and $\mathrm{P}$ in patients without and with mild to moderate ascites but not in patients with refractory ascites. As well as significant decreases in $\mathrm{Pl} R$ activity mention levels and P and RRI in patients without and with mild to moderate ascites but not in patients with refractory ascites Conclusion The administration of midodrine improves systemic haemodynamics associated with a suppression of the renin activity in cirrhotic patients without or with mild ascites. But had no effect in patients with refractory ascites

\section{P39 AN ANALYSIS OF REBLEEDING RATES FOR VARICEAL HAEMORRHAGE AT A REGIONAL CENTRE: WHAT IS THE APPLICABILITY AND POTENTIAL COST FOR EARLY TIPS?}

doi:10.1136/gutjnl-2011-300857a.39

${ }^{1} \mathrm{D}$ Harman, ${ }^{1} \mathrm{R}$ McCorry, ${ }^{2} \mathrm{~F}$ Khan, ${ }^{1} \mathrm{R}$ O'Neill, ${ }^{1} \mathrm{M}$ James, ${ }^{1} \mathrm{~S}$ Ryder, ${ }^{1} \mathrm{G}$ Aithal ${ }^{1}$ I N Guha. ${ }^{1}$ Nottingham University Hospitals NHS Trust; ${ }^{2}$ Sherwood Forest Hospitals NHS Trust

Introduction A recent randomised controlled trial demonstrated that the early use of TIPS in patients with Child-Pugh class B and C cirrhosis presenting with acute variceal haemorrhage was associated with a significant reduction in rebleeding and mortality. ${ }^{1}$ However, it remains unclear whether an additional economic benefit exists with their approach compared to the current standard of care utilising pharmacological and endoscopic therapies, and rescue TIPS. Aim We aimed to ascertain how many patients would benefit from early TIPS and the economic implications of introducing this into practice, by observing retrospective data from our tertiary care liver unit.

Method Consecutive patients admitted in 2009 with oesophageal variceal haemorrhage to a tertiary care liver unit at Nottingham University Hospitals (NUH) NHS Trust were identified retrospectively using a dedicated endoscopy database and cross-checking with the emergency medicine database. Patients with non-cirrhotic portal hypertension or isolated gastric varices were not included in our study. Standard management protocols including endoscopic therapy within $24 \mathrm{~h}$, glypressin and prophylactic antibiotics were used. Data were collected on demography, aetiology, rebleeding related hospital admissions and mortality at 12 months. Costs of rebleeding were analysed for all patients meeting inclusion criteria for the original study ${ }^{1}$ and included subsequent inpatient care costs and endoscopic/radiological intervention (figures were supplied by the NUH finance and procurement department and based on established national tariffs). The actual cost of rebleeding in our Child Pugh score 7-13 patients was compared to the theoretical cost of introducing early TIPS in this group.

Results 51 cirrhotic patients were admitted to our unit with oesophageal variceal bleeding. $20 \%$ of this cohort had Childs A, $40 \%$ Childs B and $40 \%$ Childs C cirrhosis. The rebleeding rate was $15 \%$ at 28 days and $34 \%$ at 1 -year follow-up. The survival rates were $82 \%$ at 28 days and $40 \%$ at 1 year.

35 patients (70\% of the cohort) had a Child Pugh score of 7-13. Within this subgroup there was a $31 \%$ rebleeding rate requiring 